\title{
Enhancing the production of S-adenosyl-L-methionine in Pichia pastoris GS115 by metabolic engineering
}

\author{
Ping $\mathrm{Yu}^{*}$ and Xiaoqin Shen
}

\begin{abstract}
S-adenosyl-L-methionine is an important bioactive molecule participating in a number of biochemical reactions including the transmethylation and transsulphuration reactions of proteins and the biosynthesis of aliphatic polyamines. Strategies of metabolic engineering were used to alter the metabolic flux for enhancing the production of S-adenosyl-L-methionine (SAM) in Pichia pastoris GS115. These strategies include the over-expression of Sam2 by knock-in technique and the disruption of Cbs by knock-out technique. Three strains, ZJGSU1 with knock- in of Sam2, ZJGSU2 with knock-out of Cbs and ZJGSU3 with both knock-in of Sam2 and knock -out of Cbs, were constructed for the effective production of SAM. Yields of SAM in strains ZJGSU1 and ZJGSU2 were 32- and 5-fold higher than in the original strain P. pastoris GS115, respectively. The strain ZJGSU3 had a dramatic increase in the SAM yield, and it was 46-fold higher compared to the original strain. These results indicate that there is a strong synergistic effect on the production of SAM by combining knock-in with knock-out techniques. The yield of SAM in ZJGSU3 strain was $4.37 \mathrm{~g} / \mathrm{L}$ in a $3 \mathrm{~L}$ fermentor. This study provides deep insight into the effective industrial production of SAM in future.
\end{abstract}

Keywords: S-adenosyl-L-methionine, Pichia pastoris GS115, Metabolic engineering, Fermentation

\section{Introduction}

$S$-adenosyl-L-methionine (SAM ) plays a significant role in many biological processes since it is a major methyl group donor in the transmethylation and transsulphuration reactions of proteins, nucleic acids, polysaccharides and fatty acids (Cantoni, 1953; Meister et al. 1984). SAM is very effective in the treatment of osteoarthritis, affective disorders and liver diseases (Barcelo et al. 1990; Lieber, 1999). Recently, SAM as an intracellular bioactive small molecule has deserved more attentions due to its critical roles in human health and has been successfully used in human therapy for the depressive syndrome and the osteoarthritis (Barcelo et al. 1990; Cimino et al. 1984; Osman et al. 1993).

SAM is prepared commercially by the extraction of yeast cells cultured in media supplied with L-methionine (L-Met) (Schlenk et al. 1965; Shiomi et al. 1990). Two isozymes of SAM synthase, SAM1 synthase and SAM2 synthase which are respectively encoded by genes $\operatorname{Sam} 1$

\footnotetext{
* Correspondence: yup9202@yahoo.com.cn

College of Food Science and Biotechnology, Zhejiang Gongshang University, 149 Jiaogong Road, Hangzhou 310035, Zhejiang Province, People's Republic
} of China

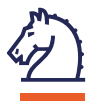

and Sam2, have been identified in Saccharomyces cerevisiae. Sam 1 transcription can be inhibited with excessive L-Met while Sam2 not (Thomas et al. 1988). S. cerevisiae has been one of the most commonly used strain for the production of SAM so far (Liu et al. 2006; Schlenk et al. 1965; Shen et al. 2008; Shiomi et al. 1990; Wang and Tan, 2008; Wang et al. 1965). However, it is not a best choice for over-expressing SAM synthase and overproducing SAM due to the presence of ethanol during culture, which often makes SAM2 ineffective and hence lowers the yield of SAM. In addition, many strains belonging to Saccharomyces sake and Kluyveromyces lactis have also been studied for the production of SAM (Mincheva et al. 2002; Shiozaki et al. 1989).

In the past twenty years, Pichia pastoris has been developed as an excellent host for the high- level expression of the heterologous gene using a strong methanolcontrolled alcohol oxidase promoter. It has a potential for the high protein expression level, the efficient secretion of products and the easy culture to a high cell density (Cerehino and Cregg, 2000). The genome sequencing of $P$. pastoris GS115 has been completed and the biosynthetic pathways of many chemicals in it have been 
elucidated (Schutter et al. 2009). In preference of the chemical or enzyme synthesis, $P$. pastoris is a better candidate to produce the high-level heterologous protein and an industrial strain used widely to produce valuable biochemical molecules (Gross et al. 1983; Matos et al. 1987; Park et al. 1996). In P. pastoris GS115, homocysteine has three alternative metabolic fates: to be remethylated to form methionine, or to be combined with serine to form cystathionine via the cystathionine- $\beta$-synthase (CBS) reaction, or to revert back to $S$ - adenosylhomocysteine (SAH) via reversal of the SAH hydrolase reaction (Figure 1) (Christopher et al. 2002; Wang et al. 2009). From viewpoint of the metabolic flux, it is possible that the over- expression of Sam will improve the accumulation of SAM. It has been confirmed by a study conducted by Li et al. (2002) in which the introduction of a pPIC3.5k-based Sam2 into P. pastoris GS115 boosted the production of SAM. Furthermore, since the cystathionine- $\beta$-synthase (CBS) acts on the removal of homocysteine from SAM cycle, the disruption of cystathionine- $\beta$ - synthase gene ( $C b s)$ is likely to lead to a high production of SAM in P. pastoris GS115. Therefore, it is very interesting to investigate whether both over-expression of Sam 2 and disruption of Cbs in P. pastoris GS115 will have a coordinative effect on the production of SAM and result in a higher yield of SAM for the industrial production.

In the present study, three genetically modified P. pastoris strains, ZJGSU1 with over- expression of Sam2, ZJGSU2 with disruption of $\mathrm{Cbs}$ and ZJGSU3 with both over-expression of Sam 2 and disruption of $\mathrm{Cbs}$, were constructed and yields of SAM in these three strains in flasks were determined and compared. Over-expression of Sam2 and disruption of Cbs in P. pastoris GS115 show a significantly synergistic effect on the production of SAM. The SAM production in ZJGSU3 strain were also investigated in a $3 \mathrm{~L}$ fermentor.

\section{Materials and methods \\ Materials}

S. cerevisiae strain (CCTCC No. M209272) was purchased from the China Center for Type Culture Collection. P. pastoris GS115 and the vectors $\mathrm{pPIC9K}$ and pPICZ $\alpha$ were purchased from the Invitrogen Co. Ltd, USA. PCR reagents, restriction endonucleases and the vector pMD19T were purchased from the TaKaRa Biotech Co. Ltd, Japan. Zeocin, SAM standard sample, G418, histidine and glutathionine were purchased from the Sigma, Co. Ltd. Multicopy Pichia Expression Kit was purchase from the Invitrogen Co. Ltd. Yeast nitrogen base and PTM1 trace metals solution were purchased from the Shanghai Sangon Bioengineering Co. Ltd, China. All other reagents were analytical grade and they were used as supplied. Primers used for the amplification of genes and their detection are listed in Table 1.

\section{Construction of the plasmid pPIC9K-Sam2 and introduction of Sam2 into $P$. pastoris GS115}

Sam2 (GeneBank accession number: gi852113) was amplified using $S$. cerevisie genome as the template and a primer set, Sam2-F and Sam2-R (Table 1). PCR products were purified and digested by $E c o \mathrm{R} \mathrm{I}$ and $B a m \mathrm{H} \mathrm{I}$, and ligated into the EcoRI-BamHI digested plasmid pPIC9K, giving the plasmid pPIC9K-Sam2 (Figure 2A). After linearized by $B g l$ II, the plasmid pPIC9K- Sam2 was transformed into P. pastoris GS115 by the electroporation method with parameters: $1.5 \mathrm{kV}, 200 \mu \mathrm{F}$ and $200 \Omega$. Transformants were screened by MD plates and G418 plates. The genomic DNA of selected transformants were isolated according to the Multicopy Pichia

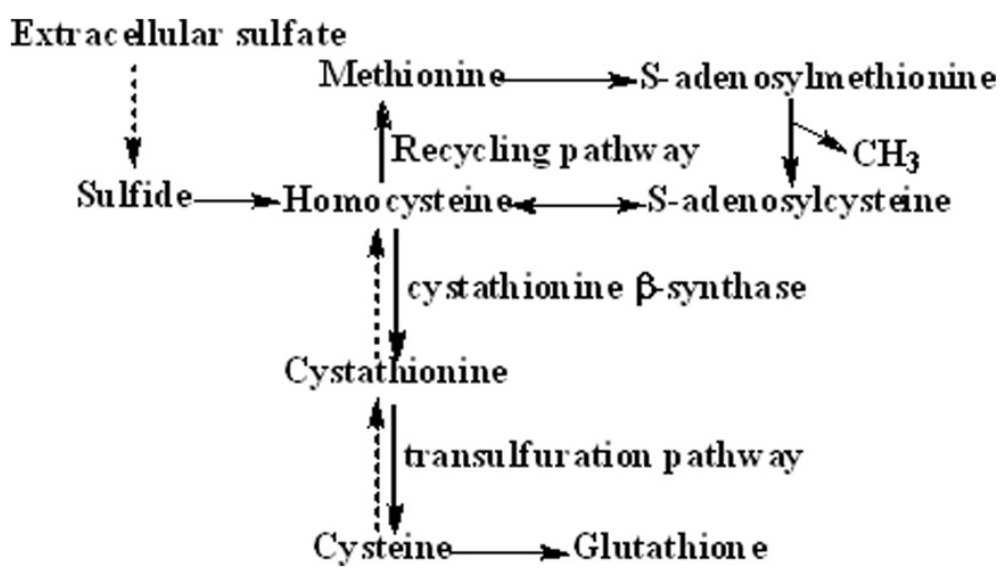

Figure 1 Metabolic network of homocysteine in P. pastoris GS115 strain. The illustration represents the homocysteine recycling and the transsulfuration pathway. Solid lines indicate reactions that are common in both yeast and mammals, whereas dotted lines are unique to yeast. 
Table 1 Oligonucleotide sequences used as primers

\begin{tabular}{ll}
\hline Names & Sequences \\
\hline Sam2-F & 5'-caggatccaccatgaccaagagcaaaact-3' $^{\prime}$ \\
\hline Sam2-R & 5'-gcggccgcgaattcagcctagcataaagaaa-3' $^{\prime}$ \\
\hline Zeocin-F & $5^{\prime}$-ggactagtagaccttcgtttgtgc-3' \\
\hline Cbs-F $R$ & $5^{\prime}$-ggactagtcggttcctggccttttg-3' \\
\hline Cbs-R & $5^{\prime}$-ttctggagcacattggaa-3' \\
\hline $1 \mathrm{~F}$ & $5^{\prime}$-agtgtatgcctagatgg-3' \\
\hline $1 \mathrm{R}$ & $5^{\prime}$-atcaaagtgctgtagttg-3' \\
\hline $2 \mathrm{~F}$ & $5^{\prime}$-acacgacctccgaccactcg-3' \\
\hline $2 \mathrm{R}$ & $5^{\prime}$-agttagacaacctgaagt-3' \\
\hline
\end{tabular}

Expression Kit provided by the manufacturer. PCRs were carried out to confirm whether Sam2 was integrated into the genomic DNA of P. pastoris GS115 strain according to the specification provided by the manufacturer.

\section{Knock-out of Cbs in P. pastoris GS115}

Cbs (GeneBank accession number: gi853059) was amplified from $P$. pastoris GS115 genome with the primer set, Cbs-F and Cbs-R (Table 2). It was then subcloned into the plasmid pMD19T to form the plasmid pMD19TCBS. The antibiotic gene of zeocin was amplified from the plasmid pPICZ $\alpha$ with the primer set, Zeocin-F and Zeocin-R (Table 2). Amplified zeocin products were digested with Spe I and subcloned into the Spe I site of the plasmid pMD19T-CBS, giving the plasmid pMD19T$\mathrm{C}-\mathrm{Z}$ (Figure 2B). The resultant pMD19T-C-Z was digested with $B g l$ II and Sal I, and the 2100 bp fragment C-Z was transformed separately into P. pastoris GS115 and ZJGSU1. MD plates supplemented with histidine, zeocin and glutathionine were used to screen transformants. Zeocin positive transformants were confirmed by PCRs with primer sets: $1 \mathrm{~F}$ and $1 \mathrm{R}, 2 \mathrm{~F}$ and $2 \mathrm{R}, 1 \mathrm{~F}$ and $2 \mathrm{R}$ (Table 2). Transformants with only disruption of $\mathrm{Cbs}$ and with both introduction of $\operatorname{Sam} 2$ and disruption of Cbs were named ZJGSU2 and ZJGSU3, respectively.

\section{P. pastoris culture for the SAM production in flasks}

Four strains, P. pastoris GS115, ZJGSU1, ZJGSU2 and ZJGSU3, were respectively inoculated into a $5 \mathrm{~mL}$ YPD medium and incubated at $30^{\circ} \mathrm{C}$ for $16 \mathrm{~h}$ as seed cultures. A $1 \mathrm{~mL}$ aliquot of the seed culture was added to $100 \mathrm{~mL}$ BMGY medium (yeast extract $1 \%$, peptone $2 \%$, glycerol $1 \%, 10 \times \mathrm{YNB} 10 \%, 0.1 \mathrm{M}$ potassium phosphate buffer, $\mathrm{pH}$ 6.0) supplemented with $1 \%$ L-Met in $500 \mathrm{~mL}$ flasks and cultured at $30^{\circ} \mathrm{C}$ for $4 \mathrm{~d}$. $1.5 \%(\mathrm{v} / \mathrm{v})$ of methanol as an inducer and carbon source was added daily for three times after one day of growth. After $96 \mathrm{~h}$ of culture, $1 \mathrm{~mL}$ of sample was taken out for determining the yield of SAM.

\section{Fermentation of the ZJGSU3 strain in a $\mathbf{3}$ L fermentor}

The ZJGSU3 strain was grown in a BMGY medium at $30^{\circ} \mathrm{C}$ for $24 \mathrm{~h}, 100 \mathrm{~mL}$ of cultures were inoculated in a 3 $\mathrm{L}$ fermentor containing $1.8 \mathrm{~L}$ of minimal salts (PTM) fermentation medium: $\mathrm{H}_{3} \mathrm{PO}_{4}$ (85\% stock), $27 \mathrm{~mL} / \mathrm{L}$; $\mathrm{CaSO}_{4}, 0.93 \mathrm{~g} / \mathrm{L} ; \mathrm{K}_{2} \mathrm{SO}_{4}, 18.2 \mathrm{~g} / \mathrm{L} ; \mathrm{MgSO}_{4} \cdot 7 \mathrm{H}_{2} \mathrm{O}, 14.9 \mathrm{~g} / \mathrm{L}$; $\mathrm{KOH}, 4.13 \mathrm{~g} / \mathrm{L}$; Glycerol, $40 \mathrm{~g} / \mathrm{L}$ and $4.4 \mathrm{~mL} / \mathrm{L}$ of PTM1 trace metals solution ( $P$. pastoris fermentation manual of the Invitrogen). Initial fermentation conditions were as follows: dissolved oxygen (DO) was maintained above $20 \%$ by the automatic control of agitation, $\mathrm{pH}$ was 5 (adjusted with ammonium hydroxide), temperature was set at $30^{\circ} \mathrm{C}$. Cells were grown until glycerol depleted completely. This was indicated by a dramatic increase in the DO to $100 \%$. Glycerol feeding was then initiated to increase the cell biomass under limited conditions: $500 \mathrm{~mL}$ of $50 \%$ glycerol containing $6 \mathrm{~mL}$ of PTM trace salts was fed at $60 \mathrm{~mL} / \mathrm{h}$. Following the depletion of glycerol, methanol feeding was initiated at a rate of $10 \mathrm{~mL} / \mathrm{h}$ and increased gradually to a final rate of $21 \mathrm{~mL} / \mathrm{h}$. Meanwhile, $500 \mathrm{~mL}$ of the saturated L-Met

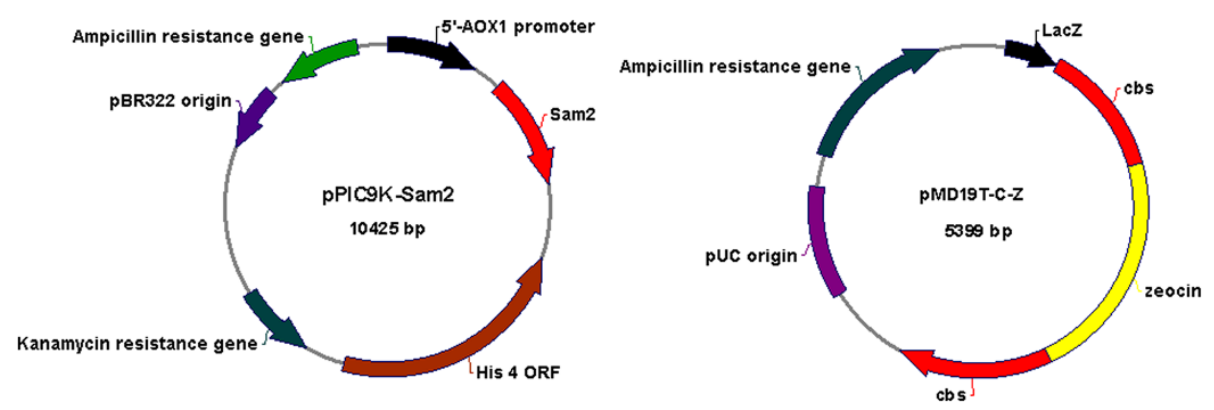

Figure 2 The maps of recombinant plasmids. (A) The map of the plasmid pPIC9K-Sam2; (B) The map of the plasmid pMD19T-C-Z. Sam 2: SAM synthase 2 gene sequence, His 4 ORF: Histidine 4 open reading frame, pBR322 origin: Origin of replication of the plasmid pPIC9K, lac Z: $\beta$-galactosidase gene $Z$, cbs: Cystathionine- $\beta$ synthase gene, pUC origin: pUC origin of replication, Zeocin: Zeocin resistance gene. 
Table 2 The activities of SAM synthase in four strains

\begin{tabular}{ll}
\hline Strains & Enzyme activity $(\mathbf{U} / \mathbf{m L})$ \\
\hline GS115 & 39 \\
\hline ZJGSU1 & 545 \\
\hline ZJGSU2 & 309 \\
\hline ZJGSU3 & 705 \\
\hline
\end{tabular}

was fed at a rate of $6 \mathrm{~mL} / \mathrm{h}$. Samples were taken out at different times for determining the yield of SAM and biomass.

\section{Determination of the yield of SAM}

The yield of SAM was determined as described by Lin et al. (2004). $1 \mathrm{~mL}$ of the sample was centrifuged at $12,000 \mathrm{rpm}$ for $10 \mathrm{~min}$, and washed twice with the deionized water, then mixed with $1 \mathrm{~mL}$ of $1.5 \mathrm{M} \mathrm{HClO}_{4}$ at $4^{\circ} \mathrm{C}$ for $1.5 \mathrm{~h}$. The supernatant was collected after centrifugation at $12,000 \mathrm{rpm}$ for $5 \mathrm{~min}$ and then went through a $0.22 \mu \mathrm{m}$ filtration membrane before analysis. A $15 \mu \mathrm{L}$ of the extracted SAM sample was injected into a high performance liquid chromatography (HPLC) system (Agilent, USA) using a C18 column (Hypersil BDS column, $4.6 \mathrm{~mm} \times 250 \mathrm{~mm}, 5 \mu \mathrm{m}$ ) with a mobile phase composed of $0.01 \mathrm{M}$ ammonium formate (pH3.0) at a flow rate of $0.8 \mathrm{~mL} / \mathrm{min}$. Peak area analysis was performed based on the standard calibration curve of SAM. Due to the instability of SAM, its $p$-toluenesulfonate salt (Sigma) was used as standard sample.

\section{Determination of wet cell weight}

$10 \mathrm{~mL}$ of the sample was taken out and centrifuged at $12,000 \mathrm{rpm}$ for $10 \mathrm{~min}$, and then washed three times with the distilled water. The wet cell weight was determined using a electronic balance.

\section{Determination of the activity of SAM synthase}

The activity of SAM synthase was determined as described by Li et al. (2002). Cells in $1 \mathrm{~mL}$ of the sample were harvested by centrifugation at $6000 \mathrm{rpm}$ for $5 \mathrm{~min}$, and washed immediately with ice-cold lytic buffer (50 $\mathrm{mM}$ potassium phosphate at $\mathrm{pH} 7.4,5 \%$ (v/v) glycerol, $5 \mathrm{mM}$ mercaptoethanol, $1 \mathrm{mM}$ EDTA), and then centrifuged at 12,000 rpm for $5 \mathrm{~min}$. Cell pellets were resuspended in $1 \mathrm{~mL}$ of the lytic buffer and disrupted by the ultrasonic treatment. The supernatant was collected by centrifugation at $12,000 \mathrm{rpm}$ for $10 \mathrm{~min}$ and used as the sample to assay the activity of SAM synthase as follows: $1 \mathrm{~mL}$ of the reaction mixture, containing $20 \mathrm{mM}$ L-Met, $20 \mathrm{mM}$ ATP, $8 \mathrm{mM}$ reduced glutathione, $20 \mathrm{mM}$ $\mathrm{MgCl}_{2}, 100 \mathrm{mM} \mathrm{KCl}, 150 \mathrm{mM}$ Tris $-\mathrm{HCl}$ and the supernatant with an appropriate concentration, was incubated at $37^{\circ} \mathrm{C}$ for 1 h. $0.5 \mathrm{~mL}$ of $20 \% \mathrm{HClO}_{4}$ was then added to the reaction mixture. The resultant precipitation was removed by centrifugation at $12,000 \mathrm{rpm}$ for $10 \mathrm{~min}$. The supernatant was used for determining the yield of SAM by the HPLC. One unit of the activity of SAM synthase was defined as the amount of enzyme required to catalyze the transformation of $1 \mu \mathrm{mol}$ of L-Met into SAM per minute at $37^{\circ} \mathrm{C}$.

\section{Results}

Identification of the introduction of Sam2 or the disruption of $C b s$ and their respective effect on the production of SAM

Over 5000 transformants grew on MD plates after the electro transformation of pPIC9K-sam 2 into $P$. pastoris GS115. Transformants having a better growth rate on MD plates were streaked on YPD plates containing different concentrations of G418 $(0.5,1.0,2.0,3.0,4.0 \mathrm{mg} / \mathrm{mL})$. 25 transformants having a faster growth rate were picked from YPD plates with G418 at a final concentration of

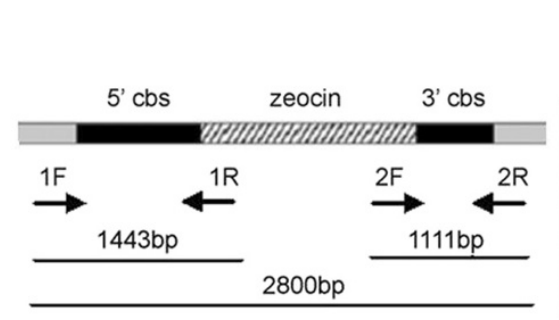

A

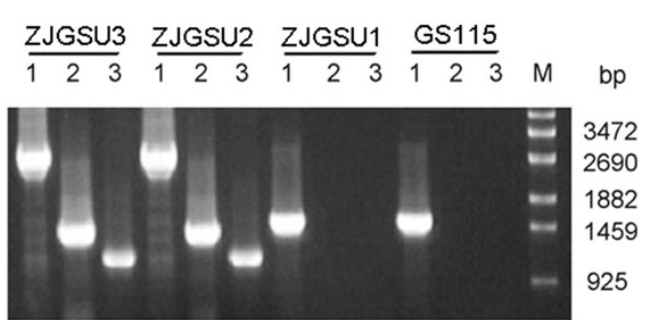

B

Figure 3 (A) The map of the C-Z gene fragment. Primers 1F, 2F, 1R and 2R were designed based on this fragment. If the homologous recombination happens, PCR product using the primer set, $1 \mathrm{~F}$ and $1 \mathrm{R}$, is a 1443-bp fragment between $5^{\prime} \mathrm{cbs}$ and zeocin (PCR 1 ). $\mathrm{PCR}$ product using the primer set, $2 \mathrm{~F}$ and $2 \mathrm{R}$, is a 1111-bp fragment between $3^{\prime} \mathrm{cbs}$ and zeocin (PCR2). PCR product using the primer set, $1 \mathrm{~F}$ and $2 \mathrm{R}$, is a 2800-bp fragment including cbs and zeocin (PCR3). (B) Identification of the Cbs disruption with PCRs. The genomic DNA was respectively extracted from different strains. The crossover of 5' was identified by PCR1, the 3'crossover was identified by PCR2, and the knock-out of Cbs by the homologous recombination was identified by PCR3. 
$4 \mathrm{mg} / \mathrm{mL}$. These transformants were cultured in flasks for evaluating the yield of SAM by HPLC. The transformant which produced the highest yield of SAM was chosen finally and named ZJGSU1. According to the manual of the "multi-copy of P. pastoris expression kit" from the Invitrogen, multiple integrated copies can lead to the increase in the $\mathrm{G} 418$ resistance level from $0.5 \mathrm{mg} / \mathrm{mL}$ (1-2 copies) to $4 \mathrm{mg} / \mathrm{mL}$ (7-12 copies). Thus, the copy number of the Sam2 gene in ZJGSU1 strain was estimated to be 712 based on its resistance to G418 at a final concentration of $4 \mathrm{mg} / \mathrm{mL}$. The yield of SAM in ZJGSU1 strain reached $0.8 \mathrm{~g} / \mathrm{L}$.

The plasmid pMD19T-C-Z was designed and constructed to disrupt $C b s$ in $P$. pastoris GS115. A knockout vector (Figure $3 \mathrm{~A}$ ) was also designed to evaluate the effect of the $C b s$ knock-out on the production of SAM. It contains a full length of $P$. pastoris $\mathrm{Cbs}$ gene (1500 bp) split by the zeocin expression cassette (1200 $\mathrm{bp}$ ) in the middle. After the plasmid pMD19T-C-Z was transformed into the host strain, the homologous recombination would occur between the genomic DNA and the transformed DNA. Recombinants could be selected by the antibiotic zeocin. Since zeocin resistance strains might have a single crossover or a random insertion of the transformed DNA, the selected transformant was confirmed by PCR using primer sets, $1 \mathrm{~F}$ and $1 \mathrm{R}, 2 \mathrm{~F}$ and $2 \mathrm{R}, 1 \mathrm{~F}$ and $2 \mathrm{R}$ (Figure $3 \mathrm{~B}$ ). PCR results demonstrated that the transformed DNA was inserted into the genomic DNA by the homologous recombination. Furthermore, since the dysfunction of $\mathrm{Cbs}$ in $\mathrm{P}$. pastoris GS115 could lead to the cysteine auxotrophy, the growth of these strains was dependent on the addition of cysteine or glutathionine to the medium. The growth of four strains, GS115, ZJGSU1, ZJGSU2 and ZJGSU3, was observed on the medium. As shown in Figure 2, four strains could grow well on the medium with glutathionine (Figure 4A), whereas strains ZJGSU2 and ZJGSU3 which have a Cbs knock-out did not on the medium

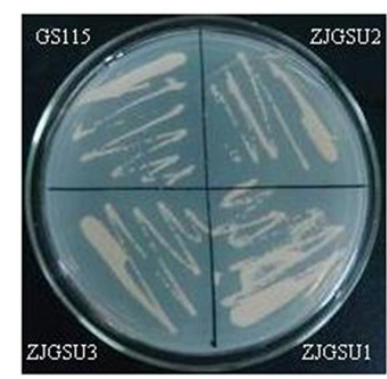

A

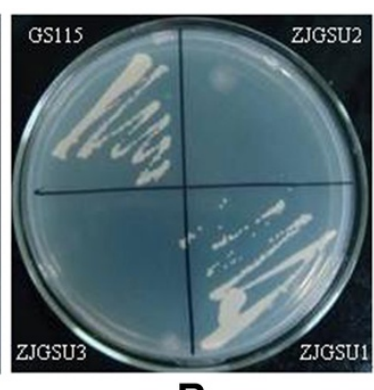

B
Figure 4 Identification of four strains on selective MD plates. (A) Strains grow on the MD plate supplemented with both histidine and glutathionine. (B) Strains grow on MD plates supplemented with histidine only.

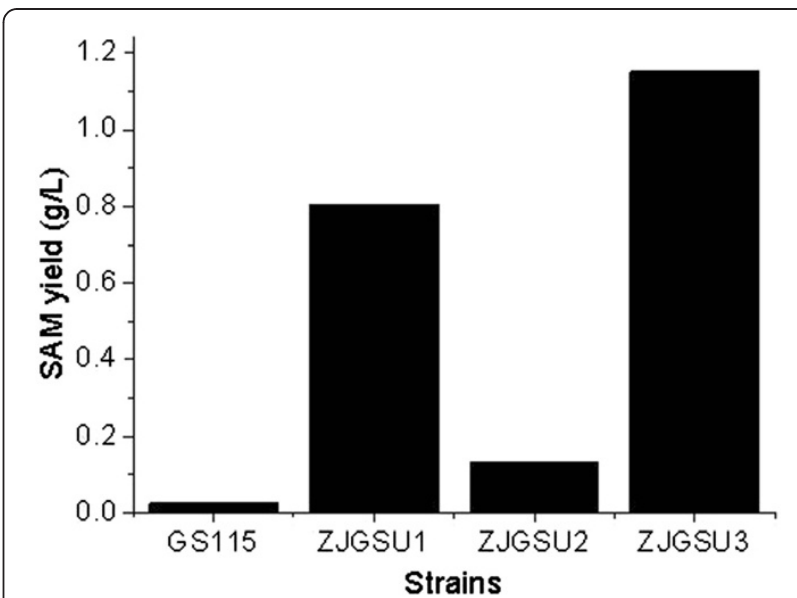

Figure $\mathbf{5}$ The yields of SAM in different strains. Data are represented as the mean of three measures. GS115: P. pastoris GS115, ZJGSU1: P. pastoris GS115 with knock- in of Sam2, ZJGSU2: $P$. pastoris GS115 with knock-out of Cbs, and ZJGSU3: $P$. pastoris GS115 with both knock-in of Sam2 and knock-out of Cbs.

without glutathionine (Figure 4B). This indicates that $\mathrm{Cbs}$ is disrupted successfully in strains ZJGSU2 and ZJGSU3. The disruption of $C b s$ also contributed to the accumulation of SAM, though not so much as the introduction of SAM synthase. The yield of SAM in the ZJGSU2 strain reached $0.13 \mathrm{~g} / \mathrm{L}$.

\section{Effect of both knock-in of Sam2 and knock-out of Cbs on the production of SAM}

Based on the above results, it is obvious that the overexpression of Sam2 or the disruption of $\mathrm{Cbs}$ has a positive effect on the production of SAM. In order to further investigate the effect of both knock-in of Sam2 and knock-out of $\mathrm{Cbs}$ on the production of SAM, the C-Z fragment from the plasmid pPMD19T-C-Z was

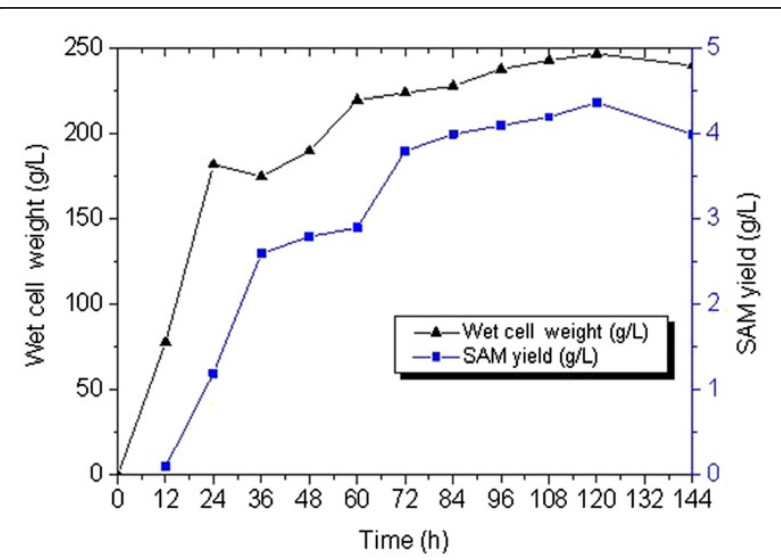

Figure 6 The growth and production curves of ZJGSU3 strain in a 3 L fermentor. $\boldsymbol{\Delta}$ : wet cell weight, $\mathbf{a}$ : the yield of SAM. The X-axis represents the time after the induction with methanol. 
transformed into the ZJGSU1 strain to produce the ZJGSU3 strain. The Cbs knock-out in ZJGSU3 strain was demonstrated by PCR analysis (Figure 3B). The yields of SAM in four strains, GS115, ZJGSU1, ZJGSU2 and ZJGSU3, are shown in Figure 5. The average yield of SAM in GS115 was $0.025 \mathrm{~g} / \mathrm{L}$, and it was $0.13 \mathrm{~g} / \mathrm{L}$ in ZJGSU2. Thus, the yield of SAM in ZJGSU2 is 5-fold higher than that in the original strain GS115. The yield of SAM in ZJGSU1 was $0.8 \mathrm{~g} / \mathrm{L}$ when $\operatorname{Sam} 2$ was introduced, 32-fold higher than in the original strain GS115. The yield of SAM in ZJGSU3 strain reached $1.2 \mathrm{~g} / \mathrm{L}$, and was over 1.4fold higher than that in ZJGSU2 and 46-fold higher than in the original strain GS115. These results indicate that both knock-in of Sam 2 and knock-out of Cbs have a synergistic effect on the enhancement of the yield of SAM.

\section{Analysis of the activity of SAM synthase}

The activities of SAM synthase in strains GS115, ZJGSU1, ZJGSU2 and ZJGSU3 were determined. The result is showed in Table 2. The activity of SAM synthase in ZJGSU3 was up to $705 \mathrm{U} / \mathrm{mL}$, and was nearly twice than that in ZJGSU2 strain and 18-fold higher than that in the original strain GS115. The activity of SAM synthase in ZJGSU1 strain was $544 \mathrm{U} / \mathrm{mL}$, 14-fold higher than that in the original strain GS115. These results further confirm that enhancing the activity of SAM synthase facilitates the production of SAM. In addition, by comparison of the activities of SAM synthase from four strains, it was also found that the knock-out of Cbs could increase the expression of Sam2, and hence enhanced the activity of SAM synthase and increased the yield of SAM.

\section{Fermentation of SAM in a $3 \mathrm{~L}$ fermentor}

Due to a favorable nutrient balance, dissolved oxygen, $\mathrm{pH}$ and high cell density in the fermentor, the production of SAM in the ZJGSU3 strain increased greatly as well as the cell growth. The host strain $P$. pastoris GS115 has an unique ability to grow on minimal media to a very high cell density with a strong tightly regulated alcohol oxidase promoter. It allows to accumulate a high level of SAM synthase, and so achieves a high yield of SAM. The glycerol was used as the only carbon source during the first two stages to stimulate the cell growth, which could further enhance the yield of SAM. At the end of the glycerol feeding stage, the wet cell weight was $182 \mathrm{~g} / \mathrm{L}$. After the depletion of glycerol, the methanol was then fed to further induce the expression of SAM synthase. By changing the methanol feeding rate, its concentration in the fermentor was maintained at a low level to minimize the toxic effect on the yeast cells. As shown in Figure 6, the maximal biomass and the highest yield of SAM were $247 \mathrm{~g} / \mathrm{L}$ and $4.37 \mathrm{~g} / \mathrm{L}$ after $96 \mathrm{~h}$ of the methanol induction, respectively.

\section{Discussion}

The results of the present study demonstrate that both introduction of $S a m 2$ and disruption of $\mathrm{Cbs}$ in P. pastoris GS115 by knock-in and knock-out techniques have a obviously synergistic effect on the production of SAM, resulting in a significant increase in the yield of SAM. The yields of SAM in ZJGSU3 strain with both introduction of Sam 2 and disruption of $\mathrm{Cbs}$ were $1.2 \mathrm{~g} / \mathrm{L}$ in flasks and $4.37 \mathrm{~g} / \mathrm{L}$ in a $3 \mathrm{~L}$ fermentor, implying that it has a commercial prospect for the large scale industrial production of SAM in future. To our knowledge, this is the first report with regard to the production of SAM by both introduction of Sam2 and disruption of $C b s$ in P. pastoris GS115 strain using the plasmid pPIC9K as the vector.

Previously, the reports pertinent to the production of SAM focused on the $S$. cerevisiae strain (Liu et al. 2006; Schlenk et al. 1965; Shen et al. 2008; Shiomi et al. 1990; Wang and Tan, 2008; Wang et al. 1965), S. sake (Shiozaki et al. 1989) and K. lacti (Mincheva et al. 2002). The traditional fermentation is a main way for the production of SAM in those strains. With the development of the genetic and metabolic engineering, enhancing yields of valuable bioactive chemicals by the modern biotechnology becomes an important trend. The yield of SAM was enhanced by altering its metabolic flux in P. pastoris GS115 strain in our study. S. cerevisiae contains two SAM synthase genes, Sam 1 and Sam2. Sam1 is repressed by the excessive L-Met, whereas $\operatorname{Sam} 2$ is not. Because L-Met is an important precursor for the effective accumulation of SAM, Sam 2 is chosen for being introduced into $P$. pastoris GS115 strain for enhancing the yield of SAM in this study. In the previous studies, Le et al. (2002) and Yu et al. (2003) reported that the knock-in of SAM synthase gene resulted in the increase in the yield of SAM in Pichia. Li et al. (2002) also reported a constructed strain with knock-out of $\mathrm{Cbs}$, but did not investigate the knock-out effect on the production of SAM. Chan and Appling (2003) reported that the deletion of $\mathrm{Cbs}$ in $\mathrm{S}$. cerevisiae did not cause a high accumulation of SAM, and the effect of the production of SAM in wild type and mutant strains was not also compared. In our study, the knock-out of $C b s$ in P. pastoris GS115 strain not only increases the yield of SAM, but also enhances the expression of SAM synthase, and so alters the metabolic flux of SAM and facilitates its further accumulation. Furthermore, a synergistic effect of both introduction of Sam2 and disruption of $C b s$ in the P. pastoris GS115 strain by knock-in and knock-out techniques on the production of SAM is for the first time reported in our study. Compared to $S$. cerevisiae, P. pastoris is a better candidate for the industrial production of recombinant proteins and valuable biochemical molecules. It can grow easily to a high cell density in a minimal salts medium with methanol as a sole carbon source and ammonium 
sulfate as a sole nitrogen source. The genetically modified P. pastoris (ZJGSU3) reaches a wet cell weight of $247 \mathrm{~g} / \mathrm{L}$ and produces $4.37 \mathrm{~g} / \mathrm{L}$ of $S A M$ in a $3 \mathrm{~L}$ fermentor.

In conclusion, a genetically modified strain ZJGSU3 for the effective production of SAM was obtained by both knock-in of Sam2 and knock-out of $\mathrm{Cbs}$ in the $P$. pastoris GS115 strain. This strain shows a great potential for the industrial production of SAM. Continuous efforts should be given to further optimize cultural conditions for the large-scale production of SAM in the fermentor and purify it for the application in the fields of biomedicines, chemical engineering and pharmaceuticals.

\section{Competing interests}

The authors declare that they have no competing interests.

\section{Acknowledgements}

We thank the National Natural Science Foundation of China (No.31171658) and the Zhejiang Province Natural Science Foundation (No.Y4080060) for generous granting this study.

Received: 24 September 2012 Accepted: 9 October 2012

Published: 30 October 2012

\section{References}

Barcelo HA, Wiemeyer JC, Sagasta CL, Macias M, Barreira JC (1990) Experimental osteoarthritis and its course when treated with S-adenosyl-L-methionine. Rev Clin Esp 187:74-78

Cantoni GL (1953) S-adenosylmethionine: a new intermediate formed enzymatically from L- methionine and adenosine triphoshate. J Biol Chem 204:403-416

Cereghino JL, Cregg JM (2000) Heterologous protein expression in the methylotrophic yeast Pichia pastoris. FEMS Microbiol Rev 24:45-66

Chan SY, Appling DR (2003) Regulation of S-adenosyl-L-methionine kk, in Sacchromyces cerevisiae. J Biol Chem 278:43051-43059

Christopher SA, Melnyk S, James SJ, Kruger WD (2002) S-adenosylhomocysteine, but not homocysteine, is toxic to yeast lacking cystathionine $\beta$-synthase. Mol Genet Metab 75:335-343

Cimino M, Vantini G, Algeri S, Curatola C, Pezzoli C, Stramentinoli G (1984) Agerelated modification of dopaminogic and beta-adrenergic receptor system: restoration to normal activity by modifying membrane fluidity with Sadenosyl-L-methionine. Life Sci 34:2029-2039

Gross A, Geresh S, Whitesides GM (1983) Enzymatic synthesis of S-adenosyl-Lmethionine from L-methionine and ATP. Appl Biochem Biotech 8:415-422

Li DY, Yu J, Tian L, Ji XS, Yuan ZY (2002) Production of SAM by recombinant Pichia pastoris. Chin J Biotech 18:295-299

Lieber CS (1999) Role of S-adenosyl-L-methionine in the treatment of liver diseases. J Hepatol 30:1155-1159

Lin JP, Tian J, You JF, Jin ZH, Xu ZN, Cen PL (2004) An effective strategy for the co- production of S-adenosyl-L-methionine and glutathione by fed-batch fermentation. Biochem Eng J 21:19-25

Liu PY, Dong HZ, Tan TW (2006) Effect of feeding pre-L-methionine on high-celldensity fermentation for S-adenosyl-L-methionine production. Chin J Biotech 22:268-272

Matos JR, Raushel F, Wong CH (1987) S-adenosyl methionine: studies on chemical and enzymatic synthesis. Biotechnol Appl Biochem 9:39-52

Meister A, Tabor CW, Tabor H (1984) Methionine adenosyltransferase (S-adenosyL- methionine synthetase) and S-adenosylmethionine decarboxylase. Adv Enzymol 56:251-282

Mincheva K, Kamburova V, Balutzov V (2002) Production of S-adenosyl-Lmethionine by a mutant strain of Kluyveromyces lactis. Biotech Lett 24:985-988

Osman E, Owen JS, Buroughs AK (1993) Review articles: a new therapeutic agent in liver disease? Aliment Pharmacol Ther 7:21-28

Park J, Tai J, Roessner CA, Scott Al (1996) Enzymatic synthesis of S-adenosyl-Lmethionine on the preparative scale. Bioorg Med Chem 4:2179-2185

Schlenk F, Zydek CR, Ehninger DJ, Dainko JL (1965) The production of S-adenosyl-Lmethionine and S-adenosyl-L-ethionine by yeast. Enzymologia 29:283-298
Schutter KD, Lin YC, Tiels P, Van Hecke A, Glinka S, Weber-Lehmann J, Rouze P, Van de Peer Y, Callewaert N (2009) Genome sequence of the recombinant protein production host Pichia pastoris. Nature Biotech 27:561-566

Shen J, Li YG, Shen GM, Yang D (2008) Fermentation modes of Saccharomyces cerevisiae HYS98 for high productivity of S-adenosyl-L-methionine. Chin J Process Eng 8:561-566

Shiomi N, Fukuda H, Fukuda Y, Murata K, Kimura A (1990) Production of Sadenosyl-L- methionine by Saccharomyces cerevisiae cells carrying a gene for ethionine resistance. Biotech Bioeng 35:1120-1124

Shiozaki S, Shimizu S, Yamada H (1989) Production of S-adenosyl-L-methionine by Saccharomyces sake: optimization of the culture conditions for the production of cells with a high S-adenosyl-L-methionine content. Agri Biol Chem 53:3269-3274

Thomas D, Rothestein R, Rosenbergn N, Surdin-Kerjan Y (1988) SAM2 encodes the second methionine S-adenosyl tansferase in Saccharomyces cerevisiae: physiology and regulation of both enzymes. Mol Cell Biol 8:5132-5139

Wang J, Tan T (2008) Pre-L-methionine feeding strategy for S-adenosyl-Lmethionine fermentative production. Chin J Biotech 24:1824-1827

Wang LZ, Zhang XQ, Li Y, Yang GX, He GY (2009) Secreted expression of Sadenosy-L- methionine synthetase in Pichia pastoris. J Agric Biotech 10:49-53

Yu ZL, Wu XJ, Li DY, Yang S, Zhou Z, Cai J, Yuan ZY (2003) Enhancement of the production of SAM by overexpression of SAM synthetase in Pichia pastoris. Acta Bioch Bioph Sin 52:127-132

doi:10.1186/2191-0855-2-57

Cite this article as: Yu and Shen: Enhancing the production of Sadenosyl-L-methionine in Pichia pastoris GS115 by metabolic engineering. $A M B$ Express 2012 2:57.

\section{Submit your manuscript to a SpringerOpen ${ }^{\odot}$ journal and benefit from:}

- Convenient online submission

- Rigorous peer review

- Immediate publication on acceptance

- Open access: articles freely available online

- High visibility within the field

- Retaining the copyright to your article

Submit your next manuscript at $>$ springeropen.com 